\title{
A POSSIBLE LINK BETWEEN THE GALACTIC CENTER HESS SOURCE AND SGR A*
}

\author{
D. R. Ballantyne ${ }^{1}$, Fulvio Melia ${ }^{1,2,3}$, Siming Liu, ${ }^{4}$ And Roland M. Crocker ${ }^{5}$ \\ Accepted to ApJ Letters
}

\begin{abstract}
Recently, HESS and other air Čerenkov telescopes have detected a source of $\mathrm{TeV} \gamma$-rays coincident with the Galactic center. It is not yet clear whether the $\gamma$-rays are produced via leptonic or hadronic processes, so it is important to consider possible acceleration sites for the charged particles which produce the $\gamma$-rays. One exciting possibility for the origin of these particles is the central black hole, Sgr A*, where the turbulent magnetic fields close to the event horizon can accelerate protons to $\mathrm{TeV}$ energies. Using a realistic model of the density distribution in a $6 \mathrm{pc} \times 6 \mathrm{pc} \times 6 \mathrm{pc}$ cube at the Galactic center, we here calculate the trajectories followed by these $\mathrm{TeV}$ protons as they gyrate through the turbulent medium surrounding Sgr A*. Diffusing out from the black hole, the protons produce $\mathrm{TeV} \gamma$-rays via $\pi^{0}$ decay following a collision with a proton in the surrounding medium. After following over 222,000 such trajectories, we find that the circumnuclear ring around $\mathrm{Sgr} \mathrm{A}^{*}$ can reproduce the observed 0.1-100 TeV HESS spectrum and flux if the protons are injected into this medium with an effective power-law index $\approx 0.75$, significantly harder than the observed photon index of 2.25 . The total energy in the steady-state $1-40 \mathrm{TeV}$ proton population surrounding Sgr A* is inferred to be $\approx 5 \times 10^{45}$ ergs. Only $31 \%$ of the emitted $1-100 \mathrm{TeV}$ protons encounter the circumnuclear torus, leaving a large flux of protons that diffuse outward to contribute to the Galactic ridge emission observed by HESS on scales of $\gtrsim 1^{\circ}$.

Subject headings: acceleration of particles — Galaxy: center — radiation mechanisms: nonthermal — gamma rays: theory
\end{abstract}

\section{INTRODUCTION}

The Galactic center is a complex environment, harboring what is believed to be a supermassive black hole, Sagittarius $\mathrm{A}^{*}\left(\operatorname{Sgr} \mathrm{A}^{*}\right)$, surrounded by a blend of new and old stellar populations, thermal and nonthermal gas components, and a wide array of both compact and diffuse high-energy sources (see Melia \& Falcke 2001 or Melia 2007 for recent reviews). In an exciting recent development, the Galactic center has been identified as a source of $\mathrm{TeV} \gamma$-rays by the air Čerenkov telescopes HESS (Aharonian et al. 2004, 2006b), Whipple (Kosack et al. 2004), CANGAROO (Tsuchiya et al. 2004) and MAGIC (Albert et al. 2006). While many of the original observations initially measured different spectra and luminosities for the Galactic center source (Aharonian et al. 2004), subsequent reprocessing and analysis has brought all the detections into agreement (Katagiri et al. 2005). Here, we concentrate exclusively on the HESS data as they provide the best constraints on the source properties.

The signal from the Galactic center was detected by HESS in observations conducted over two epochs (June-August 2003 and March-September 2004; Aharonian et al. 2004, 2006b) with a combined $\sim 38 \sigma$ excess above the background (Aharonian et al. 2006b). The spectrum of this source is a power law with photon index $2.25 \pm 0.10$, and the total flux above $1 \mathrm{TeV}$ is $(1.87 \pm 0.30) \times 10^{-8} \mathrm{~m}^{-2} \mathrm{~s}^{-1}$ (Aharonian et al. 2006b). As argued by Crocker et al. (2005), this HESS source does not appear to be coincident with the EGRET Galactic center source 3EG J1746-2851. There

\footnotetext{
${ }^{1}$ Department of Physics, The University of Arizona, 1118 East 4th Street Tucson, AZ 85721; drb, melia@ physics.arizona.edu

${ }^{2}$ Steward Observatory, The University of Arizona, 933 N. Cherry Avenue, Tucson, AZ 85721

${ }^{3}$ Sir Thomas Lyle Fellow and Miegunyah Fellow.

${ }^{4}$ Theoretical Division, Los Alamos National Laboratory, P.O. Box 1663 , Los Alamos, NM 87545; liusm@lanl.gov

${ }^{5}$ School of Chemistry and Physics, The University of Adelaide, Adelaide, South Australia, 5005 Australia; roland.crocker@adelaide.edu.au
}

are two reasons for this: first, the EGRET source excludes the Galactic center at the $99.9 \%$ confidence level (Hooper \& Dingus 2002; Pohl 2005). In contrast, the HESS source is coincident within $\sim 30^{\prime \prime}$ of $\mathrm{Sgr} \mathrm{A}^{*}$, though with a centroid displaced roughly $7^{\prime \prime}(\sim 0.4 \mathrm{pc})$ to the East of the Galactic center (Aharonian et al. 2006b). Second, the EGRET spectrum extrapolated into the HESS energy range over-predicts (by a factor $\sim 20$ ) the $\mathrm{TeV} \gamma$-ray flux of the Galactic center source. Although it is possible that both sources are associated with the SNR Sagittarius A East, the HESS TeV source is probably associated with the black hole itself, given its angular proximity to Sgr A*.

If the $\mathrm{TeV} \gamma$-rays do originate from $\mathrm{Sgr} \mathrm{A}^{*}$, then this presents somewhat of a theoretical puzzle: given what we understand about Sgr $\mathrm{A}^{*}$ and the interactions with its nearby environment, this object should not be directly producing a significant flux of $\mathrm{TeV}$ photons. Several possible scenarios for producing these $\gamma$-rays have been proposed (e.g., Atovan \& Dermer 2004; Aharonian \& Neronov 2005a b; Hinton \& Aharonian 2006), including that of Liu et al. (2006). These last authors show that protons can be energized to $\mathrm{TeV}$ energies by stochastic acceleration in a magnetically dominated funnel close to the black hole $(\sim$ $20-30$ Schwarzschild radii). The proton acceleration models of Becker et al. (2006) may also be active (or even dominant) in Sgr A*. In either case, the relativistic protons will then diffuse along magnetic fields from small scales to large radii without experiencing adiabatic loss as is the case for ejections of plasma blobs. As the protons diffuse out through the surrounding medium, they may scatter with hydrogen nuclei in a shocked stellar wind region, and with the circumnuclear disk surrounding the black hole. Such pp scattering events produce $\pi^{0}$ 's, which subsequently decay into two photons. Thus, this model suggests that protons random-walking their way into the gaseous few central parsecs surrounding Sgr A* may ultimately be the source of the HESS-detected $\mathrm{TeV} \gamma$-rays (e.g., Aharonian \& Neronov 2005b). It is this process that we aim 
to examine carefully in this Letter by carrying out a highlydetailed analysis of the proton transport and interaction, starting at the acceleration site near the black hole and extending out as far as several parsecs. We assume a distance to the Galactic center of $R_{0}=7.94 \mathrm{kpc}$ (Eisenhauer et al. 2003).

\section{CALCULATIONS}

\subsection{Outward Proton Diffusion from $\operatorname{Sgr} A$ *}

The motion of a relativistic proton moving through the ISM is governed solely by the Lorentz force due to the interstellar magnetic field: $d \mathbf{v} / d t=(\mathbf{v} \times \mathbf{\Omega}) / \gamma$, where $\gamma$ is the Lorentz factor for a proton with velocity $\mathbf{v}$ and $\boldsymbol{\Omega} \equiv e \mathbf{B} / m c$ is the gyrofrequency for a particle with charge $e$ and mass $m$ under the influence of a magnetic field B. Thus, given a description for the magnetic field in the computational volume, the trajectory of the proton can be followed exactly. As there is no net electric field, the magnitude of the velocity $v_{0}$ remains constant.

Proton trajectories are calculated in a $6 \mathrm{pc} \times 6 \mathrm{pc} \times 6 \mathrm{pc}$ cube consisting of $10^{6}$ equally spaced cells centered on the Galactic center. The hydrogen density $n_{\mathrm{H}}$ within this volume is taken from the results of simulations by Rockefeller et al. (2004). These authors computed the density distribution in this region caused by the interactions of stellar winds from the surrounding young stars. In addition to the stellar wind gas, the volume also contains a high-density 'torus' with an inner radius of $1.2 \mathrm{pc}$ and a thickness of $1 \mathrm{pc}$ representing the observed circumnuclear disk containing molecular gas.

The magnetic field is assumed to be generated everywhere in this region with an intensity that is proportional to $n_{\mathrm{H}}$. The average density in the model stellar-wind gas (taken to be any region where $n_{\mathrm{H}}<3 \times 10^{3} \mathrm{~cm}^{-3}$ ) is $\left\langle n_{\mathrm{H}}^{\mathrm{sw}}\right\rangle=121 \mathrm{~cm}^{-3}$, while it is $\left\langle n_{\mathrm{H}}^{\mathrm{mt}}\right\rangle=233,222 \mathrm{~cm}^{-3}$ within the model molecular torus. Taking $k T=1.3 \mathrm{keV}$ as the average temperature of the stellar-wind gas (Baganoff et al. 2003; Rockefeller et al. 2004), $100 \mathrm{~K}$ for the temperature of the molecular torus (Rockefeller et al. 2004 and references therein), and assuming equipartition, the average field intensity $B_{0}$ is $\approx 3 \mathrm{mG}$ in the stellar-wind region and $\approx 0.35 \mathrm{mG}$ within the torus. As the proton moves through different densities in the computational domain, $B_{0}$ is scaled to the appropriate value, indicated by the local gas density.

While the above procedure scales the magnetic field intensity at any position in the grid, we also require a physical description of the field direction at each point. A useful prescription for describing a turbulent magnetic field was developed by Giacalone \& Jokipii (1994) to analyze the fundamental physics of ionic motion in the ISM (see also Kowalenko \& Melia 2000). This quasi-analytic approach assumes that the magnetic field fluctuations are static and follow a Kolmogorov spectrum (though in principle any type of turbulence may be modeled in this way). At every position in the grid, the three-dimensional magnetic field is written as the sum over a given number of transverse waves of random polarization, with a wavevector oriented in a random direction. In contrast to Giacalone \& Jokipii (1994), we add together fluctuations from 200 wavelengths, ranging from $0.1 v_{0} / \Omega_{0}$ to $10 v_{0} / \Omega_{0}$, where $\Omega_{0}$ is the proton gyrofrequency at $B_{0}$. In this fashion, we may then generate all three components of the magnetic field as functions of the position coordinates, for each proton released from the origin with initial velocity $\mathbf{v}_{0}$ in a random direction.

With the above ingredients, the trajectory of each proton can be calculated exactly as it traverses the volume around Sgr $\mathrm{A}^{*}$. The calculation is completed once the proton leaves the computational domain. Since the observed-frame time step is proportional to $\gamma^{2}$, computational resources limited the calculations to proton energies between 1 and $100 \mathrm{TeV}$. A total of 222,617 proton trajectories were calculated with energies uniformly distributed in this range. Figure 1 shows a typical trajectory calculated in this fashion.

\subsection{The Pion Production Rate and Gamma-ray Emissivity}

As the proton random-walks its way toward the edge of the calculation domain by scattering off the turbulent magnetic field, it may collide with a low-energy proton in the ambient medium and produce pions via the reaction $p p \rightarrow p p \pi^{0} \pi^{ \pm}$, where a variable number of pions are produced to conserve charge. The neutral pion will subsequently decay into two photons. In principle, one or more of the two protons in each scattering event may retain (or gain) sufficient energy to produce additional pions in subsequent interactions, but we will ignore their contribution to the overall pion production rate for this application. In addition, a charge exchange may occur (roughly $1 / 4$ of the time), in which a neutron is produced in the exit channel. A fitting formula for the cross-section of ppscattering is given by Eidelman et al. (2004), and is typically $\sigma_{p p} \sim 40$ mbarns for proton energies between 1 and $10 \mathrm{TeV}$.

To compute the fraction of relativistic protons that undergo pp-scattering, we first split the trajectories into 21 energy bins $(\log (E / \mathrm{eV})=12,12.1, \ldots, 13.9,14)$. Each proton path is then followed and if the particle enters a volume element with $n_{\mathrm{H}}>3 \times 10^{3} \mathrm{~cm}^{-3}$ (these regions will dominate the $\gamma$-ray emissivity) the incremental optical depth $d \tau=\bar{n}_{\mathrm{H}} \sigma_{p p}(E) d l$ to pp-scattering within this grid cell is calculated. Here, $d l$ is the distance the proton traveled since the previous time step $(\approx 1 / \Omega)$ and $\bar{n}_{\mathrm{H}}$ is the average density encountered by the proton as it moved a distance $d l$. As long as the particle remains in that cell, we continue to sum $d \tau$. If the proton scatters out of the volume element and then subsequently returns, the new values of $d \tau$ are added to the previous total. This procedure is followed for each proton trajectory in each of the 21 energy bins. The fraction of protons at energy $E$ that undergo a pp-scattering event in a volume element with $n_{\mathrm{H}}>3 \times 10^{3} \mathrm{~cm}^{-3}$ is $f(E)=1-e^{-\left(\int d \tau\right) / N}$, where $N$ is the number of calculated trajectories at energy bin $E$.

A power-law spectrum of proton energies in the relativistic regime is a natural consequence of many acceleration processes (e.g., Liu et al.2006), but the exact value of the spectral index depends on the details of how the protons escape. As we shall see below, the rate of diffusion and subsequent scattering of the protons is highly energy dependent. Therefore, we treat the "injected" spectral index as a variable to be fixed by the fitting procedure. By "injected" we mean the distribution of protons leaving $\mathrm{Sgr} \mathrm{A}^{*}$ 's region of influence and entering the wind-shocked region surrounding it.

The proton spectrum is thus written as $d n / d E_{\text {inj }}=$ $K\left(E / E_{\min }\right)^{-\alpha}$, where $K \propto \mathcal{E}$, the total energy in protons in ergs between $E_{\min }=1 \mathrm{TeV}$ and $40 \mathrm{TeV}$. The spectrum of scattered protons in a grid cell is thus $d n / d E=$ $\left(d n / d E_{\text {inj }}\right) f(E)$, where $f(E)$, defined above, is the fraction of protons with energy $E$ which collide with an ambient proton in that volume element. However, $f(E)$ is only known down to $\log E=12$, so $d n / d E$ is extrapolated to $0.1 \mathrm{TeV}$ using the chosen power-law index $\alpha$ and the value of $f(E)$ at $\log E=12$. As $\gamma$-rays at a given energy are preferen- 

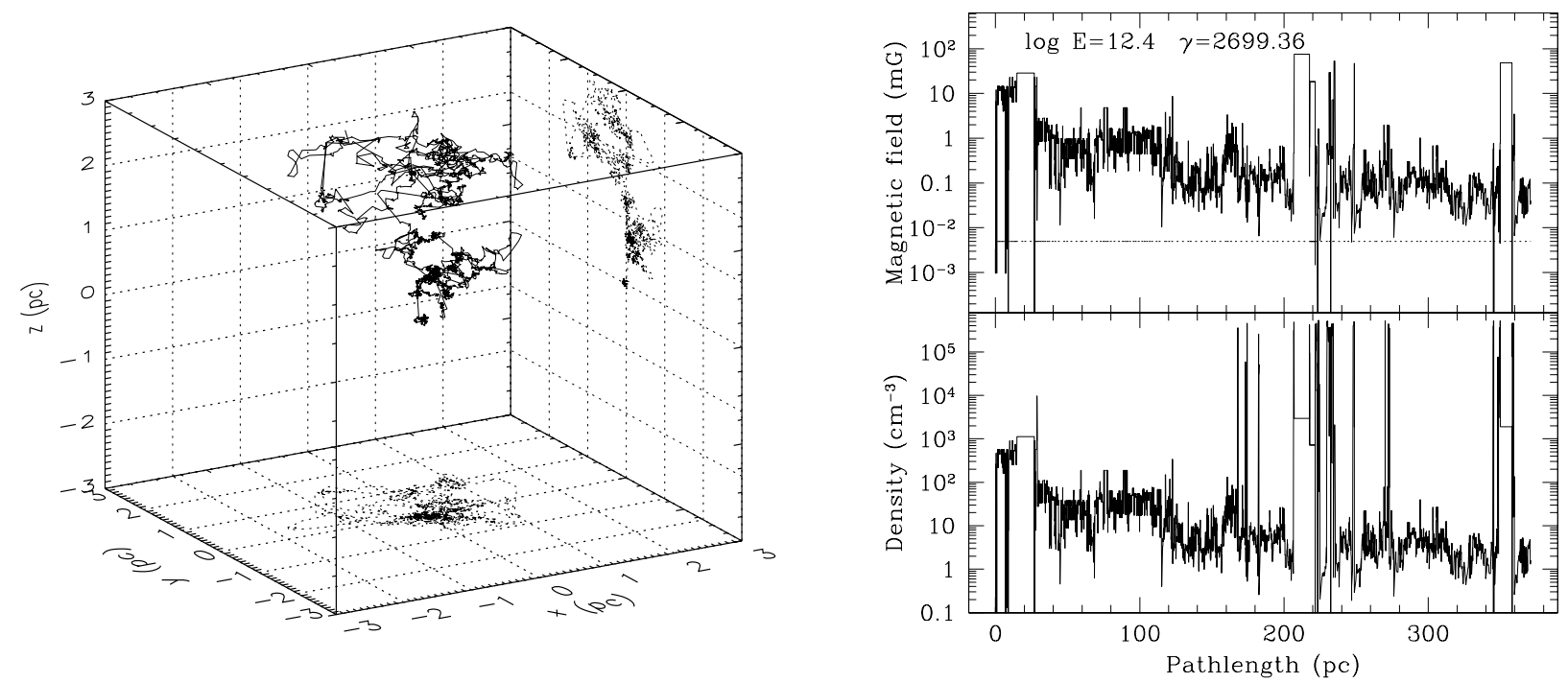

FIG. 1.- (Left) Complete trajectory of a $\log (E / \mathrm{eV})=12.4$ proton from ejection at $\mathrm{Sgr} \mathrm{A}^{*}$ (the origin) until it escapes the computational volume at $z=3 \mathrm{pc}$. The $\mathrm{x}-\mathrm{y}$ plane is the sky plane. (Right) The magnetic field strength and gas density encountered by this proton on its trajectory. The particle traveled for 1,211 yrs and traversed $371.3 \mathrm{pc}$ on its journey outward from Sgr A*. It spent most of its time in the lower-density stellar wind gas but did have numerous encounters with the high density molecular torus. The dotted line in the upper-panel indicates the lowest magnetic field strength for which the Lorentz equation was solved. For the extremely low density zones which had magnetic field strengths below this limit the particle was moved in a straight line until it encountered higher density gas.

tially produced by protons with $\sim 10 \times$ greater energy, this extrapolation will not greatly affect the predictions between 0.1 and $1 \mathrm{TeV}$. The fraction of protons that interact with the circumnuclear torus becomes very small at energies greater than $100 \mathrm{TeV}$ (see $\S$ 3), so it is not necessary to extrapolate $d n / d E$ beyond $100 \mathrm{TeV}$. Now armed with the $d n / d E$ profile within each cell, we calculate the $\pi^{0}$ and $\gamma$-ray emissivity for different values of $\mathcal{E}$ and $\alpha$ using the formalism described by Crocker et al. (2005). The emissivities are then converted into fluxes before the values are summed to produce the predicted $\gamma$-ray spectrum from $0.1-100 \mathrm{TeV}$.

\section{RESULTS}

We compared the model spectra to the observed 2003 and 2004 HESS data between 0.3 and $30 \mathrm{TeV}$ (taken from Aharonian et al. 2004, 2006b) using $\chi^{2}$ fitting. The lowest $\chi^{2}$ was found for $\mathcal{E}=5 \times 10^{45}$ ergs and $\alpha=0.75$ $\left[\chi^{2} /\right.$ d.o.f $=41.7 / 31(29.1 / 31)$ using the smaller (larger) of the observed error bars, and d.o.f. $=$ degrees of freedom]. This value for the spectral index is very robust with $\Delta \chi^{2} \approx+5$ for $\alpha=0.7$ or 0.8 . The predicted $0.1-100 \mathrm{TeV}$ spectrum and 1-10 TeV image of the Galactic center with these parameters are shown in Fig. 2. The expected 1-10 TeV luminosity from this spectrum is $4.7 \times 10^{34} \mathrm{erg} \mathrm{s}^{-1}$. The predicted $1-$ $100 \mathrm{TeV}$ flux is $1.83 \times 10^{-8} \mathrm{~m}^{-2} \mathrm{~s}^{-1}$, in good agreement with the observed value of $(1.87 \pm 0.30) \times 10^{-8} \mathrm{~m}^{-2} \mathrm{~s}^{-1}$ (Aharonian et al. 2006b).

The fact that the best fit corresponds to $\alpha=0.75$ indicates that the spectrum of injected particles must be significantly harder than the observed $\mathrm{TeV} \gamma$-ray spectrum (for which the spectral index is $2.25 \pm 0.10$ ). This is simply because the higher energy protons diffuse outward from the Galactic center faster than the low energy ones and as a result traverse, on average, a much smaller path length. These particles are then less likely to encounter the molecular torus and undergo a pp-scattering event that will produce a $\mathrm{TeV} \gamma$-ray. A harder intrinsic spectrum is therefore required to compensate for this deficit and account for the observed spectral shape. The de- rived value of $\alpha=0.75$ is much harder than the "standard" power law with index $\sim 2-2.5$ that one might expect from simple first-order Fermi scattering. Perhaps this may be taken as an indication that $\mathrm{Sgr} \mathrm{A}^{*}$ is not the source of energetic hadrons producing the $\gamma$-ray glow in its vicinity. Alternatively, the intrinsic $\alpha \sim 2$ proton spectrum may have been altered by interactions much closer to the black hole. Another possibility is that the required hard proton spectrum might be an indication that stochastic acceleration continues as the particles diffuse outwards from the initial injection region. Future work will investigate the origin and viability of the hard proton spectrum.

We find that only about $30 \%$ of the computed trajectories encounter the circumnuclear torus; the rest travel unimpeded to larger distances. However, this fraction is highly energy dependent: $\sim 73 \%$ of the emitted protons encounter the torus at $\log (E / \mathrm{eV})=12-12.4$, but this drops to $47 \%$ at $\log (E / \mathrm{eV})=13$, and finally to $5 \%$ at $\log (E / \mathrm{eV})=14$. As a result, the predicted $\gamma$-ray spectrum exhibits a roll-over at energies $\gtrsim 20 \mathrm{TeV}$ (Fig. 2). This is a robust prediction of models where $\mathrm{Sgr} \mathrm{A}^{*}$ is the source of the relativistic protons and can be tested with future HESS observations.

The energy-dependent proton interaction rate also suggests that a significant flux of $\mathrm{TeV}$ protons escapes from the inner few pc surrounding Sgr A* to interact with more distant molecular gas. This would be consistent with the observed emission from the Galactic center ridge. Aharonian et al. (2006a) report that the inner $200 \mathrm{pc}$ of the Galaxy glows in $\mathrm{TeV} \gamma$-rays, with the dominant emission correlated with the distribution of dense molecular gas. In addition, the spectrum of the Galactic ridge emission is observed to be close to that of the Galactic center source. The best-fitting model for the Galactic center HESS source predicts an energy of $\mathcal{E}=5 \times 10^{45}$ ergs in protons between 1 and $40 \mathrm{TeV}$. Aharonian et al. (2006a) estimate that a hadron distribution with an energy $\sim 10^{49} \mathrm{ergs}$ (from $4-40 \mathrm{TeV}$ ) is required to produce the observed Galactic ridge emission. The ridge region is much larger than the volume within which the Galac- 

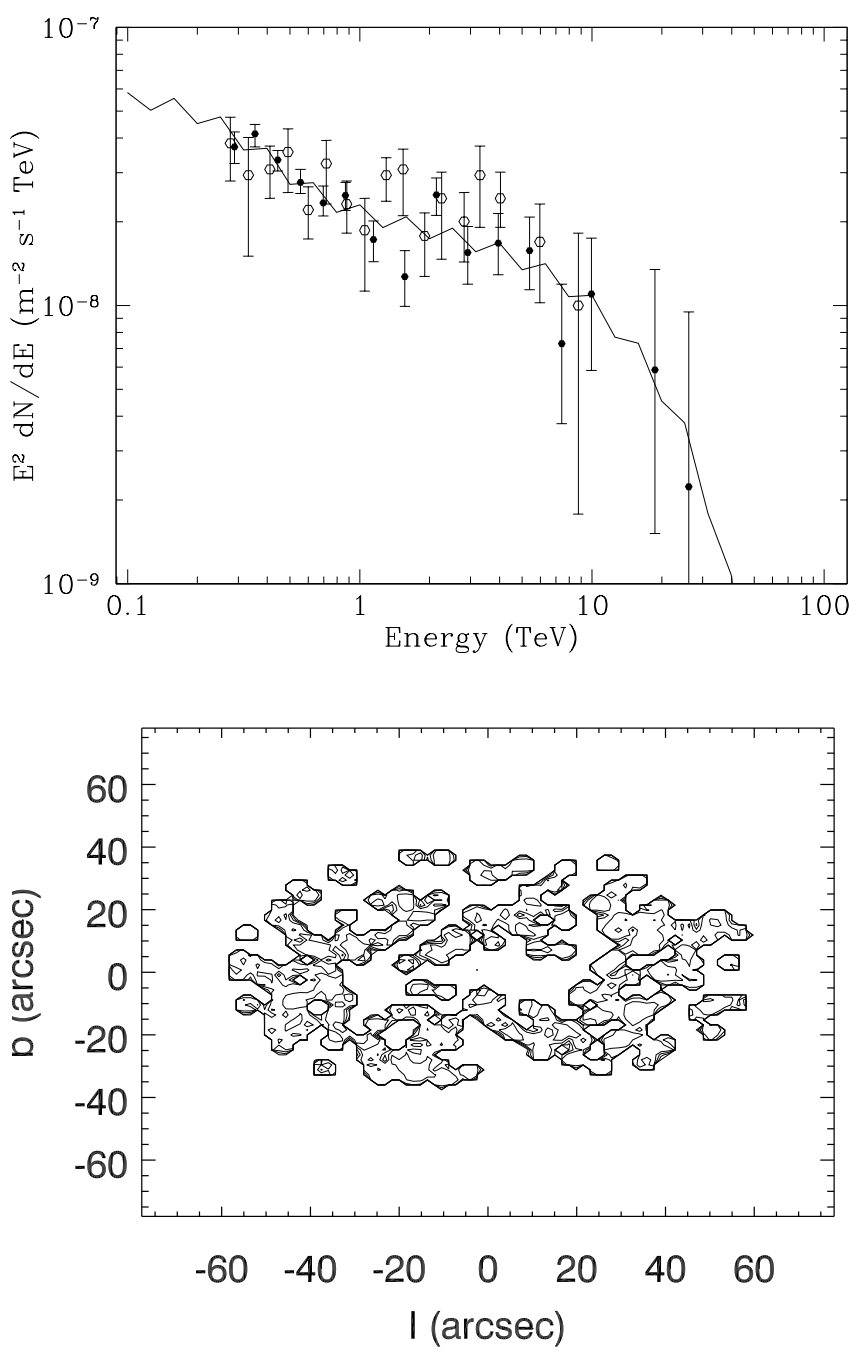

FIG. 2.- (Top) The solid line is the predicted spectrum from the Galactic center region due to pp-scattering. The total energy required in protons between 1 and $40 \mathrm{TeV}$ is $5 \times 10^{45} \mathrm{ergs}$, and the injected spectrum is $d n / d E_{\text {inj }} \propto E^{-0.75}$. The open data points are the 2003 observed values from HESS taken from Aharonian et al. (2004), while the solid points are from the 2004 HESS observations (Aharonian et al. 2006b). (Bottom) Contours of 1-10 TeV flux predicted from the same model that produced the spectrum in the left-hand panel. The contours are at $\log f=$ $-15,-14,-13,-12,-11,-10$, and $-9\left[\mathrm{~s}^{-1} \mathrm{~m}^{-2}\right]$. The oblateness evident in this image is due to the tilt angle of the molecular torus. Sgr A* is at the origin.

tic center $\mathrm{TeV}$ source is produced, so the escaping protons would build up the much larger energy content over time. The diffusion time to reach such large distances is $>10^{4}$ years (Aharonian et al. 2006a), so the particle accelerator would have to be at least that old.
Our simulations show that an injected proton index $\alpha \approx$ 0.75 is required to produce the observed $\gamma$-ray index $\alpha_{\text {obs }} \approx$ 2.25 , a steepening of $3 / 2$. Homogeneous diffusion with a Kolmogorov or Bohm turbulence spectrum would produce a steepening of $1 / 3$ and 1 , respectively. Our treatment in this paper has followed the diffusion of protons taking into account the variable 3-D gas density and magnetic field surrounding Sgr A*. On the other hand, diffusion calculations assume a homogeneous medium, which produces misleading results when applied to regions as complex as that near Sgr $A^{*}$. As such, our finding that a steepening of $3 / 2$ is required, instead of $\sim 1$, may be traceable to our more accurate treatment of inhomogeneity.

\section{CONCLUSIONS}

The HESS observations suggest that there may be a common cause for both the Galactic-center point source and the diffuse emission along the ridge. TeV protons accelerated near Sgr A* can account for the observed flux and spectrum of the central source through scattering events with ambient protons within the circumnuclear ring. The proton spectrum injected into the gas surrounding $\mathrm{Sgr} \mathrm{A}^{*}$ must have a hard power-law index of 0.75 , implying a non-standard acceleration process or interactions close to the acceleration region. Our simulations have resulted in a predicted $1-10 \mathrm{TeV}$ image of the source for comparison with future high-resolution observations. We have found, in particular, that the predicted $\mathrm{TeV}$ image mirrors closely the structure of the torus, including an oblateness due to projection effects in the plane of the sky. The total energy required in protons between 1 and $40 \mathrm{TeV}$ is $5 \times 10^{45}$ ergs and a significant fraction of the protons do escape the neighborhood of Sgr A* without undergoing a pp collision; these presumably diffuse to much larger distances where they can interact with other molecular material, possibly accounting for the observed Galactic ridge emission. Although future calculations are required to confirm this suggestion, and other origins for the observed HESS source are possible (e.g., Wang et al. 2006; Hinton \& Aharonian 2006), this work supports the view that Sgr A* may be an important site for particle acceleration at the center of the Galaxy.

We thank the anonymous referee for very useful comments. DRB is supported by the University of Arizona Theoretical Astrophysics Program Prize Postdoctoral Fellowship. This work was funded in part at the University of Arizona by NSF grant AST-0402502, and has made use of NASA's Astrophysics Data System Abstract Service. FM is grateful to the University of Melbourne for its support (through a Sir Thomas Lyle Fellowship and a Miegunyah Fellowship).

\section{REFERENCES}

Aharonian, F.A. \& Neronov, A. 2005a, ApJ, 619, 306

Aharonian, F.A. \& Neronov, A. 2005b, Ap\&SS, 300, 255

Aharonian, F.A. et al., 2004, A\&A, 425, 13

Aharonian, F.A. et al., 2006a, Nature, 439, 695

Aharonian, F.A. et al., 2006b, Phys. Rev. Lett., 97, 221102

Albert, J. et al., 2006, ApJ, 638, L101

Atoyan, A. \& Dermer, C.D., 2004, ApJ, 617, L123

Baganoff, F.K. et al., 2003, ApJ, 591, 891

Becker, P.A., Le, T. \& Dermer, C.D., 2006, ApJ, 647, 539

Crocker, R.M., Fatuzzo, M., Jokipii, J.R., Melia, F. \& Volkas, R.R. 2005, ApJ, 622,892

Eidelman, S. et al., 2004, Phys. Lett. B., 592, 1
Eisenhauer, F., Schödel, R., Genzel, R., Ott, T., Tecza, M., Abuter, R., Eckart, A. \& Alexander, T., ApJ, 597, L121

Giacalone, J., \& Jokipii, J.R. 1994, ApJ, 430, L137

Hinton, J.A. \& Aharonian, F.A., 2006, ApJ, in press (astro-ph/0607557)

Hooper, D. \& Dingus, B. 2002, in the proceedings of the 34th COSPAR

Scientific Assembly (astro-ph/0212509)

Katagiri, H. et al., 2005, ApJ, 619, L163

Kosack, K. et al., 2004, ApJ, 608, L97

Kowalenko, V., \& Melia, F. 2000, MNRAS, 310, 1053

Liu, S., Petrosian, V., \& Melia, F. 2004, ApJ, 611, L10

Liu, S., Melia, F., Petrosian, V. \& Fatuzzo, M. 2006, ApJ, 647, 1099 
Melia, F., 2007, The Galactic Supermassive Black Hole, Princeton University Press

Melia, F. \& Falcke, H. 2001, ARA\&A, 39, 309

Pohl, M. 2005, ApJ, 626, 174

Rockefeller, G., Fryer, C. L., Melia, F. \& Warren, M. S. 2004, ApJ, 604, 662
Tsuchiya, K. et al., 2004, ApJ, 606, L115

Wang, Q.D., Lu, F.J. \& Gotthelf, E.V., 2006, MNRAS, 367, 937 